\title{
O PAPEL DA ENFERMAGEM FRENTE À PROMOÇÃO DA SAÚDE DE CRIANÇAS QUE FREQUENTAM CRECHES: uma revisão integrativa
}

\author{
Nadja Myrelly de Medeiros ALENCAR ${ }^{1}$ \\ Maria Benegelania PINTO² \\ Luciana Dantas Farias de ANDRADE ${ }^{3}$ \\ Heloisy Alves de MEDEIROS ${ }^{4}$ \\ Édija Anália Rodrigues de LIMA ${ }^{5}$ \\ Nathanielly Cristina Carvalho de Brito SANTOS ${ }^{6}$
}

\begin{abstract}
${ }^{1}$ Enfermeira pela Universidade Federal de Campina Grande (UFCG), Campus Cuité. Cuité, PB, Brasil. E-mail: nadjamedeiros01@hotmail.com.

${ }^{2}$ Enfermeira. Mestre. Docente do Curso de Enfermagem do Centro Acadêmico de Vitória da Universidade Federal de Pernambuco - CAV/UFPE. E-mail:benegelania@yahoo.com.br

${ }^{3}$ Enfermeira. Doutora em Psicologia. Professora da Unidade Acadêmica de Enfermagem - UAENFE, Universidade

Federal de Campina Grande - UFCG, Cuité-PB, Brasil. E-mail:luciana.dantas.farias@gmail.com

${ }^{4}$ Enfermeira.Doutoranda em Enfermagem pela UFMG. Docente da UAENFE, UFCG, Cuité - PB.E-mail:

heloisymedeiros@hotmail.com

${ }^{5}$ Enfermeira.Mestre em Enfermagem pela Universidade Federal da Paraíba - UFPB. Docente da UAENFE, UFCG,

Cuité - PB. E-mail: edijaprof@ hotmail.com

${ }^{6}$ Enfermeira. Especialista em Serviços de Saúde Pública. Doutoranda do Programa de Pós-Graduação em Enfermagem

da Universidade Federal da Paraíba (UFPB). Professora do Curso de Bacharelado em Enfermagem da Universidade

Federal de Campina Grande (UFCG). Campus Cuité. Cuité, PB, Brasil. E-mail: nathaniellycristina@ hotmail.com.
\end{abstract}

\section{Recebido em: 13/04/2016 - Aprovado em: 21/11/2017 - Disponibilizado em: 30/12/2017}

\begin{abstract}
RESUMO
Objetivou-se sumarizar o conhecimento científico produzido entre os anos 2009 e 2015 acerca do papel da enfermagem frente à promoção da saúde de crianças em creches. Trata-se de uma revisão integrativa da literatura, realizada em abril de 2015, a partir das bases de dados MEDLINE, LILACS, BDENF e a biblioteca eletrônica SCIELO, utilizando os descritores "Saúde da criança AND Enfermagem $A N D$ Creche" e "Saúde da criança AND Enfermagem. A amostra foi composta por sete artigos.A análise foi realizada utilizando-se a técnica de analise de conteúdo, a partir da qual os achados foram elencados três categorias temáticas, que apontaram a relevância da atuação do enfermeiro diante das dificuldades apresentadas pelos pais para conduzirem as crianças até os serviços de saúde para consulta de puericultura; bem comopara educação dos pais frente às dúvidas e tabus sobre o cuidado com a saúde das crianças e;ainda, perante a falta de preparo dos profissionais da creche para promoção da saúde da criança. Percebe-se que a inserção do enfermeiro na creche ainda é heterogêneaEspera-se que estudo venha despertar e fortalecer a parceria entre profissionais de saúde e educação, para a melhoria na qualidade da atenção primária à saúde da criança, família e comunidade com foco na integralidade em consonância com o Sistema Único de Saúde.
\end{abstract}

Palavras-chave: Saúde da criança. Enfermagem. Creche.

\begin{abstract}
This study aimed to summarize the scientific knowledge produced between the years 2009 and 2015 on the role of the nursing health promotion of children in day care. This is an integrative literature review, held in April 2015, from the MEDLINE, LILACS, BDENF and electronic library Scientific Electronic Library Online (SciELO) using the keywords "Health AND Nursing AND Creche child "and" Health aND nursing child. The sample consisted of seven articles. The analysis was performed using the content analysis technique, from which the findings were listed three thematic categories, which pointed out the relevance of the nurse's performance on the difficulties faced by parents to drive children to the health services for childcare consultation; as well as education of parents facing doubts and taboos about the care of children's health and; yet, given the lack of preparation of the nursery for child health promotion professionals. It is noticed that the insertion of the nurse in the nursery is still heterogeneous expected to study will awaken and strengthen the partnership between health and education professionals, to improve the quality of primary health care of the child, family and community focused on completeness in line with the National Health System.
\end{abstract}

Keywords: Child health. Nursing.Daycare. 


\section{INTRODUÇÃO}

Durante muito tempo a criança teve seus cuidados sob a inteira responsabilidade da família, e em especial da mãe, o que contribuía para a formação de vínculo entre estes(VASCONSCELOS;

TANCREDI; MARIN, 2013).Com as mudanças socioeconômicas do século XIX, as mulheres ganharam espaço no mercado de trabalho, e os filhos passaram a frequentar creches e vivenciar a maior parte do tempo longe do domicílio e desse cuidado familiar.

No entanto, apesar dessa instituição assumir o papel de assistência integral, fatores como à aglomeração de crianças e a imaturidade do sistema imunológico própria do processo de crescimento infantil podem contribuir para o adoecimento desse grupo, sendoas doenças infecciosas as mais prevalentes(VASCONSCELOS; TANCREDI; MARIN， 2013; PEDRAZA; QUEIROZ; SALES, 2014).

No Brasil,de acordo com dados do Departamento de Informática do Sistema Único de Saúde do Brasil (DATASUS) (2010),no ano de 2009, entre as doenças responsáveisporinternações hospitalares de crianças menores de cinco anos de idade no Sistema Único de Saúde (SUS) estão às relacionadas ao aparelho respiratório,as infecciosas e parasitárias, e asdo aparelho digestivo. Contudo, entre os menores de 1 ano de idade prevalece as infeções respiratórias, enquanto na faixa de 1 a 4 anos,hámaior proporção de doenças infecciosas e parasitárias, e do aparelho digestivo.

Diante disso, o Ministério da Saúde tem investido em políticas públicas visando promover uma atenção integral e de qualidade à criança, como destaca a Política Nacional de Atenção Integral à Saúde da Criança (PNAISC) recentemente publicado sob a portaria 1.130/2015(BRASIL, 2015).

Nessa perspectiva a atuação da Enfermagem na atenção a criança no cenário da creche é indispensável, visto que pode contribuir de forma significativa por meio das ações de vigilância em saúde, realizando intervenções de identificação e controle das condições de saúde, orientando familiares e educadores e possibilitando um cuidado integral na primeira infância(ESTEVES et al, 2012). Ante as considerações apresentadas o presente estudo tem como objetivo analisar o conhecimento científico acerca do papel da enfermagem frente àpromoção da saúde de crianças que frequentam creches.

\section{MÉTODO}

O presente estudo consiste em uma revisão integrativa da literatura científica, norteada pela seguinte questão: Qual o conhecimento científico produzido acerca do papel da enfermagem frente à promoção da saúde de crianças que frequentam creches? Foi realizada por meiodas seguintes etapas: 1elaboração da questão norteadora da pesquisa; 2- estabelecimento da amostragem ou busca na literatura pela fixação dos critérios de 
inclusão e exclusão dos estudos, as bases de dados e o método de seleção dos estudos; 3definição das informações a serem extraídas dos estudos selecionados ou categorização dos estudos; 4- avaliação dos estudos incluídos na revisão integrativa nos quais se realizou a análise dos dados; 5- interpretação dos resultados; 6- apresentação da revisão/síntese do conhecimento(BOTELHO; CUNHA; MACEDO, 2011).

Dessa forma, a busca e a seleção dos artigos foram realizadasem abril de 2015pelo pesquisador principal e replicado por um segundo pesquisador, para maior fidedignidade, não sendo identificadas divergências na seleção. Para tanto foram utilizadas as bases de dados: Medline (Medical LiteratureAnalysisandRetrieval System Online), Lilacs (Literatura LatinoAmericana e do Caribe em Ciências da Saúde),BDENF (Base de Dados de Enfermagem) e a biblioteca eletrônica Scielo (Scientific Eletronic Library Online)aplicando-se os descritores "saúde da criança, enfermagem e creche"cadastrados nosDescritores em Ciências da Saúde (DECS). Para aumentar o escopo de busca, esses foram cruzados entre si com o uso do operador booleano “ $A N D$ ”.

Foram adotados como critérios de inclusão: estudo que versasse sobre o papel da enfermagem frente à promoção da saúde de crianças que frequentam creches; publicações nacionais e internacionais; estar nos idiomas português, inglês e espanhol, ter tempo de publicação de janeiro de 2009 a abril de 2015; disponibilizados na íntegra e publicados em periódicos de Enfermagem. Foram excluídos os artigos repetidos, indisponíveis na íntegra, além de monografias e teses.

Foram identificados285 artigos, dos quais 80 foram selecionados para leitura na íntegra. Destes, 71 foram excluídos por fuga do tema,dois por duplicidade nas bases de dados, e um por indisponibilidade na íntegra, restando sete artigos para análise.

Para caracterizaçãodos estudos, utilizou-seum instrumento validadopor Ursi (2005), que adaptado contemploutítulo do estudo, autor, periódico, ano da publicação, região do estudo, objetivos, características metodológicas, resultados e conclusões. Os dados foram analisados por meio da análise de conteúdo que abrange três etapas: préanálise; exploração do material por meio de recortes das partes representativas dos artigos; tratamento dos resultados obtidos e interpretação(MINAYO, 2012).

A partir disso, os principais aspectos acerca do papel da enfermagem frente à promoção da saúde de crianças que frequentam creches, foram abordados em três categorias temáticas, e discutidos a partir da literatura pertinente.

\section{RESULTADOS}

Conforme a tabela 1 verifica-se que entre os sete artigos selecionados, predominou publicações datadas de 2010 a 

qualitativa, realizados nas regiões $\mathrm{Sul}$ e nacionais.

Tabela 1 - Distribuição dos artigos considerando bases de dados ou biblioteca eletrônica, autor, ano, periódico, tipo e local do estudo e principais resultados. Cuité-PB, 2015.

\begin{tabular}{|c|c|c|c|c|}
\hline $\begin{array}{l}\text { Base de } \\
\text { dados } \\
\text { ou } \\
\text { Biblioteca } \\
\text { Eletrônica } \\
\end{array}$ & $\begin{array}{l}\text { Autor } \\
\text { /Ano }\end{array}$ & Periódico & $\begin{array}{l}\text { Tipo/Local e } \\
\text { Região do } \\
\text { estudo }\end{array}$ & Principais Resultados \\
\hline BDENF & $\begin{array}{l}\text { GabaniFL, } \\
\text { MaebaraC } \\
\text { ML, } \\
\text { Ferrari } \\
\text { RAP; } 2010\end{array}$ & $\begin{array}{l}\text { Revista de } \\
\text { Enfermagem } \\
\text { Escola Anna } \\
\text { Nery }\end{array}$ & $\begin{array}{l}\text { Estudo } \\
\text { descritivo } \\
\text { transversal, } \\
\text { Londrina - PR, } \\
\text { Região Sul }\end{array}$ & $\begin{array}{l}\text { Categoria 2: Para os trabalhadores dos Centros de } \\
\text { Educação Infantil (CEI) entre as práticas realizadas } \\
\text { prevalecem a catação }(42,4 \%) \text {, uso do pente fino } \\
(34,4 \%) \text { e pouco mais de } 10 \% \text { consideram a } \\
\text { educação dos familiares e higiene pessoal importante. } \\
\text { Como principais dificuldades estão falta de } \\
\text { colaboração dos pais, ambiente desfavorável, } \\
\text { desconhecimento acerca do tratamento e falta de } \\
\text { apoio dos profissionais de saúde. } \\
\text { Categoria 3: Como práticas realizadas identificou-se } \\
\text { como prevalentes a catação e o uso do pente fino } \\
\text { (42,4\%), uso de medicamentos (34,4\%) e pouco mais } \\
\text { de } 10 \% \text { consideravam a educação dos familiares e } \\
\text { crianças e melhora da higiene pessoal. As principais } \\
\text { dúvidas estão relacionadas ao tratamento e o ciclo de } \\
\text { vida do parasito, e a maior dificuldade é a falta de } \\
\text { colaboração dos pais }(81,3 \%) \text {, ambiente desfavorável } \\
\text { (aglomeração de crianças) }(11,9 \%) \text {, desconhecimento } \\
\text { do tratamento correto }(5,1 \%) \text { e falta de apoio dos } \\
\text { profissionais de saúde }(1,7 \%) \text {. }\end{array}$ \\
\hline BDENF & $\begin{array}{c}\text { Cortez } \\
\text { DN, Pio } \\
\text { DF, Silva, } \\
\text { MJ, Lívio } \\
\text { PF; } 2012\end{array}$ & $\begin{array}{l}\text { Revista de } \\
\text { Enfermagem } \\
\text { do Centro } \\
\text { Oeste Mineiro }\end{array}$ & $\begin{array}{c}\text { Estudo } \\
\text { qualitativo,Mina } \\
\text { s Gerais-MG, } \\
\text { Região Sudeste }\end{array}$ & $\begin{array}{l}\text { Categoria 1: Os pais apontam a presença do } \\
\text { profissional enfermeiro na creche como aquele } \\
\text { responsável pela assistência à saúde da criança, } \\
\text { considerando a importância do acompanhamento do } \\
\text { crescimento e desenvolvimento para detecção de } \\
\text { doenças e agravos. }\end{array}$ \\
\hline BDENF & $\begin{array}{l}\text { Carneiro } \\
\text { JM, Brito } \\
\text { APB, } \\
\text { Santos } \\
\text { MEA; } \\
2011\end{array}$ & $\begin{array}{l}\text { Reme: Revista } \\
\text { Mineira de } \\
\text { Enfermagem }\end{array}$ & $\begin{array}{c}\text { Estudo } \\
\text { descritivo de } \\
\text { abordagemquant } \\
\text { itativa- Coronel } \\
\text { Fabriciano, Vale } \\
\text { do Aço-MG, } \\
\text { Região Sudeste }\end{array}$ & $\begin{array}{l}\text { Categoria 1: } 88 \% \text { das crianças apresentaram } \\
\text { resultado "Normal"; } 12 \% \text { desenvolvimento "Suspeito } \\
\text { ou duvidoso" e nenhuma apresentou"Anormal" no } \\
\text { setor da linguagem. }\end{array}$ \\
\hline BDENF & $\begin{array}{l}\text { Faria ML, } \\
\text { Wichr P; } \\
2014\end{array}$ & $\begin{array}{l}\text { Reme: Revista } \\
\text { Mineira de } \\
\text { Enfermagem }\end{array}$ & $\begin{array}{c}\text { Estudo } \\
\text { descritivo e } \\
\text { analítico, com } \\
\text { abordagem } \\
\text { qualitativa, } \\
\text { Diamantina- } \\
\text { MG, Região } \\
\text { Sudeste }\end{array}$ & $\begin{array}{l}\text { Categoria 2: Os educadores das creches atribuem o } \\
\text { adoecimento das crianças a determinantes externos à } \\
\text { instituição; a doenças da infância e a determinantes } \\
\text { próprios da instituição. A assistência prestada à } \\
\text { criança doente é baseada nos conhecimentos das } \\
\text { educadoras e em caso de necessidade levam a criança } \\
\text { para a unidade de saúde e avisam à família. } \\
\text { Categoria 3: Não existe um programa específico de } \\
\text { capacitação em saúde para os professores de } \\
\text { educação. }\end{array}$ \\
\hline LILACS & $\begin{array}{l}\text { Motta JA, } \\
\text { Silva PO, } \\
\text { Marta CB, } \\
\text { Araújo }\end{array}$ & $\begin{array}{l}\text { Revista } \\
\text { Enfermagem } \\
\text { UERJ }\end{array}$ & $\begin{array}{c}\text { Estudo } \\
\text { descritivo de } \\
\text { abordagem } \\
\text { qualitativa, São }\end{array}$ & $\begin{array}{l}\text { Categoria 1: Cuidados integrados: para os } \\
\text { profissionais as necessidades consideradas mais } \\
\text { importantes foram alimentação, higiene, sono, parte } \\
\text { pedagógica e cuidado afetivo. }\end{array}$ \\
\hline
\end{tabular}




\begin{tabular}{|c|c|c|c|c|}
\hline & $\begin{array}{c}\text { BBM, } \\
\text { Francisco } \\
\text { MTR, } \\
\text { Junior } \\
\text { HCS; 2012 }\end{array}$ & & $\begin{array}{c}\text { Pedro da } \\
\text { Aldeia- RJ, } \\
\text { Região Sudeste }\end{array}$ & $\begin{array}{l}\text { Categoria 2: Os profissionais da creche entendem } \\
\text { que a presença do enfermeiro traria benefícios às } \\
\text { crianças por meio de ações que auxiliariam na } \\
\text { prevenção e promoção de saúde. } \\
\text { Categoria 3: As ações de prevenção das doenças } \\
\text { infectocontagiosas, envolvem somente a higiene } \\
\text { bucal, e que a incidência poderia ser diminuída com a } \\
\text { ação efetiva de profissionais da saúde para o processo } \\
\text { de capacitação dos profissionais da creche, e por não } \\
\text { haver esta capacitação para ações de saúde e não } \\
\text { possuírem uma visão ampliada de saúde, os } \\
\text { profissionais da creche se sentem incapazes de } \\
\text { desenvolver alguma ação de saúde com as crianças. }\end{array}$ \\
\hline SciELO & $\begin{array}{l}\text { Monteiro } \\
\text { FPM, } \\
\text { Caetano } \\
\text { JA, Araújo } \\
\text { TL; } 2010\end{array}$ & $\begin{array}{l}\text { Escola Anna } \\
\text { Nery: Revista } \\
\quad \text { de } \\
\text { Enfermagem }\end{array}$ & $\begin{array}{l}\text { Estudo } \\
\text { bibliográfico }\end{array}$ & $\begin{array}{l}\text { Categoria 1: Avaliação nutricional como prática do } \\
\text { cuidado de enfermagem mediante acompanhamento } \\
\text { do crescimento e desenvolvimento infantil por meio } \\
\text { dados e índices antropométricos como método } \\
\text { diagnóstico, no contexto hospitalar e/ou na saúde } \\
\text { pública; além da identificação de fatores } \\
\text { determinantes da nutrição da criança como } \\
\text { alimentação e ambiente. }\end{array}$ \\
\hline SciELO & $\begin{array}{c}\text { Sarubbi } \\
\text { JV, } \\
\text { Muylaert } \\
\text { CJ, Gallo } \\
\text { SM, Gallo } \\
\text { PR; } 2014\end{array}$ & $\begin{array}{l}\text { Revista da } \\
\text { Escola de } \\
\text { Enfermagem } \\
\text { da USP }\end{array}$ & $\begin{array}{l}\text { Estudo } \\
\text { transversal, de } \\
\text { caráter } \\
\text { descritivo e de } \\
\text { abordagem } \\
\text { qualitativa, } \\
\text { São Paulo-SP, } \\
\text { Região Sudeste }\end{array}$ & $\begin{array}{l}\text { Categoria 2: Ao se inserirem nas creches, os } \\
\text { profissionais de enfermagem relatam o desafio de } \\
\text { redimensionar seu conhecimento de forma a repensar } \\
\text { as ações de cuidado, destacam que ao realizar um } \\
\text { trabalho vinculado à educação em saúde, estão } \\
\text { atuando sobremaneira como um educador na } \\
\text { interface de conhecimentos entre o campo da saúde e } \\
\text { da educação.Para os profissionais a experiência com } \\
\text { a criança é percebida como transformadora, a partir } \\
\text { das vivências que passam a ter com elas e do vínculo } \\
\text { que estabelecem. }\end{array}$ \\
\hline
\end{tabular}

\section{DISCUSSÃO}

Considerando o tempo de permanência da criança nacrecheé premente a atuação do enfermeiro para promoção da saúde e integralidade do cuidado infantil. Nesse sentido, apesar do número reduzido de artigos identificados nesta revisão, pode-se sugerir que a temática vem ganhando espaço entre os pesquisadores preocupados com a saúde da criança na contemporaneidade, uma vez que as publicações estavam distribuídas nos últimos cinco anos.

Porém, o fato de a maioria dos estudos terem sido realizados na região Sul do país, pode indicar que esse interesse pela temática ainda é heterogêneo, e isso pode contribuir para desfechos negativos como o adoecimento e morte de crianças ainda na primeira infância por doenças preveníveis.

\section{Categoria 1: Acompanhamento do} crescimento e desenvolvimento infantil na creche

A atuação dos profissionais de enfermagem no cuidado à criança frenteàs suas peculiaridades no crescimento e desenvolvimento, abrange a implementação de ações preventivas, curativas e de promoção da saúde realizadas nas Unidades de Estratégia de Saúde da Família (CORTEZ et al, 2012;CARNEIRO; BRITO; SANTOS, 
2011; MONTEIRO; CAETANO; ARAÚJO, 2010;MOTTA et al, 2012)

$\mathrm{Na}$ revisão em telaé possível perceber que as ações e práticas do enfermeiro frente à saúde da criança também devem ser realizadasno âmbito da creche com oacompanhamento do crescimento e desenvolvimento infantil. Estudo (CORTEZ et al, 2012) evidenciou que a relevância deste acompanhamento no âmbito da creche ocorre devido ao fato dequando realizado apenas nas Unidades Básicas de Saúde (UBS),pode não ter continuidadedevido à distância até a unidade de saúde, à jornada de trabalho dos pais, bem como porestes não considerarem importante esse tipo de atendimento à criança.

Por outro lado, a percepção dos pais quanto ao acompanhamento do crescimento e desenvolvimento, de alguma forma, apresenta pontos satisfatórios, destacando a preocupação em relação ao estado de saúde, do desenvolvimento, dos sinais e sintomas de acometimento ou agravos à saúde, conhecendo a importância deste acompanhamento para a vida de seus filhos(CORTEZ et al, 2012).

Assim,o reconhecimentopelos responsáveis da criança acerca da importância do acompanhamento infantil pode contribuir para evitar complicações e agravos à saúde das crianças, obtendo ainda diminuição de custos com hospitalizações desnecessárias(FROTA et al, 2011), pois possibilita o seguimento adequado conforme preconiza o Ministério da Saúde de acordo com a faixa etária (BRASIL, 2012).

No que diz respeito ao desenvolvimento infantil de crianças em creches e os possíveis desvios identificados a partir deste acompanhamento, estudo(CARNEIRO; BRITO; SANTOS, 2011),permitiu identificar crianças com desenvolvimento da linguagem "suspeito ou duvidoso". Coadunando este achado, outro estudoevidenciou a relevância deste seguimento para identificação precoce de possíveis alterações nesse processo, visto que das crianças que apresentaram alteração na aquisição e desenvolvimento da linguagem, quatro tinham um distúrbio severo (SANTOS; LEMOS; LAMOUNIER, 2010).

Frente a isso, o acompanhamento sistemático de crianças que frequentam creches, realizado por profissionais de saúde qualificados, vem subsidiar a atenção prestada nos serviços de saúde, podendo contribuir para redução dos casos de problemas de saúde de uma parte significativa desta população(SOUZA et al, 2013). Ademais, reforça as diretrizes de cuidado à criança na atenção primária à saúde quando apresenta que deve ser realizado em qualquer ambiente a qual a criança esteja inserida, incluindo à creche(MURTA et al, 2011).

Como fatores determinantes da nutrição infantil, a alimentação e o ambiente foram mencionados em estudo(MONTEIRO; CAETANO; ARAÚJO, 2010) como 
características quepodem influenciar no crescimento e desenvolvimento de criança saudáveis e que devem ser valorizados na avaliação da criança pelo profissional de saúde.

Estudo que avalioucrianças em umacreche em Belo Horizonte, Minas Geraisevidenciou que um número expressivo delas apresentou bom estado nutricional, o que pode estar relacionado ao papel das instituições na oferta de uma alimentação adequada e de qualidade, minimizando os riscos nutricionais(SANTOS; LEMOS; LAMOUNIER, 2010).

Contudo, limitações biológicas, ambientais e sociais que possam existir no contexto da creche podem representar fatores de vulnerabilidade para alterações no crescimento e desenvolvimento infantil(SANTOS; LEMOS; LAMOUNIER, 2010).Assim, exigem uma avaliação sistemática e contínua da criança por um profissional de saúde.

Isso aponta para a necessidade da interação entre serviço de saúde e de educação para a continuidade do acompanhamento infantil no cenário da creche. Para tanto, a atuação do profissional enfermeiro nessas instituições buscafortalecer as ações sensibilização dos responsáveis das crianças enfatizando a importância do seguimento infantil e a promoção de um crescimento saudável.

\section{Categoria 2: Ações de educação em saúde} para familiares de crianças que

\section{frequentam creches}

Os principais resultados da pesquisa destacam a importância daatuação do profissionalde enfermagem nasações de educação em saúde voltadaspara os familiares de crianças que frequentam creches, visto seu papel de educador (MOTTAet al, 2012; GABANI; MAEBARA; FERRARI, 2010; FARIA; WICHR, 2014; SURUBBI et al, 2014).

Estudorealizado nos centros de educação infantil do município de Londrina Paranádemonstraque os pais têm influência no combate a pediculose, pois a falta de colaboração dos mesmos dificulta o controle, sendo apontado por $10 \%$ dos profissionais da creche a educação dos familiares e criançasalém da melhora da higiene pessoalcomo fundamentais para diminuição desta ectoparasitose (GABANI; MAEBARA; FERRARI, 2010).

Chama a atenção o fato dea maioria dos pais não considerarem a pediculose doença, e que seria apenas diante de uma infestação(GOLDSCHMIDT; LORETO, 2012). Essa informação é preocupante visto que alimitações de compreensão dos pais quanto a estaectoparasitose ser considerada doença, dificulta sua prevenção, o que torna necessária a realizaçãodas ações educativas pelos profissionais de saúde para todos os familiares envolvidos nesse contexto, no 
sentido de esclarecer dúvidas e possibilitar melhor compreensão, bem como sensibilizálos acerca dos cuidados para combater à pediculose(GABANI;

MAEBARA;

FERRARI, 2010).

Os profissionais de saúde ao se inserirem no âmbito das instituições de ensino infantil relatam o desafio das ações de cuidado, e ainda destacam que ao desempenharem um trabalho associado à educação em saúde, estão agindo como um educador na interface de conhecimentos entre o campo da saúde e da educação(SURUBBI et al, 2014).

Percebe-se assim, que, a inclusão de um profissional de saúde na creche não diminui as responsabilidades da unidade básica de saúde, mas auxilia na promoção da saúde através de medidas como avaliação da caderneta de saúde da criança e promoção da educação em saúde(FARIA; WICHR, 2014).

Logo, percebe-se a necessidade de haver orientações para familiares de crianças inseridas no contexto institucional da creche, pois é por meio de ações educativas, que os pais e/ou responsáveis tomam conhecimento dos devidos cuidados com seus filhos, utilizando condutas que podem contribuir para prevenir agravos asaúde das crianças nas creches.

Assim, reforça a importância da integração entre setores de educação e de saúde, compartilhando ações e responsabilidades frente aos eixos de atenção à saúde da criança.

\section{Categoria 3: Realização de capacitação acerca da prevenção em saúde para profissionais de educação que atuam em creches.}

Os principaisestudosrevelam a necessidade da realização de capacitações de profissionais de educação para prevenção em saúde de crianças que frequentam creche(MOTTA et al, 2012; GABANI; MAEBARA; FERRARI, 2010; FARIA; WICHR, 2014).

Esse achado reflete a fragilidade da atuação dos profissionais de saúde voltadas para os profissionais de educação na promoção da saúde de crianças inseridas neste contexto, que apesar das potencialidades para o cuidado à criança, também apresentam aspectos de vulnerabilidade que demandam o olhar atento dos profissionais de educação.

Frente a isto, ressalta-se que são várias as dúvidas e desconhecimento que os educadores de creches carregam consigo a respeito do cuidado com a criança institucionalizada. A esse respeito, estudo (GABANI; MAEBARA; FERRARI, 2010,demonstrou fragilidades no conhecimento destes profissionais em relação a pediculose, visto que a maioria acredita que piolhos ou ectoparasitas pulam e se prolifera mais frequentemente no verão e gostam de sangue doce. Em relação ao tratamento, os funcionários consideram eficaz o uso de 
substâncias de uso doméstico como sal, vinagre, e outras inflamáveis e tóxicas como querosene e inseticida, além disso, o shampoo comum é considerado o mais útil para a pediculose.

Outro estudodestaca que para os profissionais deuma creche municipal noRio de Janeiro, a ação preventiva para as parasitoses intestinais envolve apenas a higiene bucal (MOTTA et al, 2012). Este achado é alarmante, pois paraFernandes; Barbosa (2011), as condutas para prevenção desse parasita se devem por meio de ações como, manuseio e preparo apropriados com a alimentação, uso de calçados, tratamento da água, saneamento básico e uso de medicamentos, bem como programas educacionais voltados à higiene.

SegundoMotta et al (2012), revela que os educadores da creche afirmaram que não são realizadas ações de promoção da saúde, desconhecendo que os mesmos podem promover a saúde das crianças e, que essas ações podem ser realizadas apenas por profissionais de saúde. Neste contexto, é de competência de o profissional da educação conhecer e saber orientar quanto às doenças mais presentes no seu ambiente de trabalho, destacando que este não irá assumir mais uma responsabilidade no seu dia a dia, mas, deverão ser capacitados no que se refere a estes conhecimentos(GOLDSCHMIDT; LORETO, 2012).
Em contrapartida, estudoaponta que não há capacitação específica em saúde para os profissionais da educação (FARIA; WICHR, 2014). ComplementaMottaet al (2012), que os educadores inseridos na sua pesquisa afirmaram que por não existir a capacitação, os mesmos sentem-se incapazes de desenvolver ações de saúde com as crianças e reconhecem a importância deste treinamento para que possa proporcionar um melhor cuidado, evitando a ocorrência de incidentes no ambiente de trabalho.

A capacitação dos profissionais da educação pode apresentar impacto na promoção e recuperação da saúde das crianças, proporcionando um cuidado integral no ambiente de educação, com maior suporte intersetorial (FARIA; WICHR, 2014). Assim, considerando a permanência das crianças maior parte do tempo na creche, reforça a importância do treinamento em saúde para os educadores, permitindo a promoção da saúde e a interação entre educar e cuidar.

\section{CONCLUSÃO}

Neste estudo, foi possível perceber a importância da atuação do enfermeiro no cenário da creche, na perspectiva de pais e educadores. Isso se deve ao fato de os pais não disporem de tempo para acompanharem suas crianças às unidades de saúde, apresentaram limitações de conhecimento e tabus que dificultam a implementação de medidas de prevenção, bem como pela falta 
de preparo dos profissionais que atuam nas instituições de ensino frente ao cuidado da criança, que ocorre embasado em conhecimentos populares.

Contudo, pode-se perceber que a inserção deste profissional na instituição infantil ainda não se constitui uma realidade comum. Em função disso, acredita-se que este estudo venha contribuir para a atenção à saúdeda criança diante da promoção da saúde pela enfermagem nas instituições de educação, de modo a despertar e fortalecer a parceria entre profissionais de saúde e educação, para a melhoria na qualidade da atenção primária à saúde da criança, família e

\section{REFERÊNCIAS}

BOTELHO, L. L. R.; CUNHA, C. C. A.; MACEDO, M. O método da revisão integrativa nos estudos organizacionais. Revista Eletrônica Gestão e Sociedade, Belo Horizonte, v. 5, n. 11, p. 121-136, mai/ago. 2011.

BRASIL, Ministério da Saúde. Portaria ${ }^{\circ}$ 1.130 , de 5 de agosto de 2015. Política Nacional de Atenção Integral à Saúde da Criança (PNAISC) no âmbito do Sistema Único de Saúde (SUS).

BRASIL. Ministério da Saúde. Saúde da criança: crescimento e desenvolvimento. Brasília: Ministério da Saúde, 2012. (Cadernos de Atenção Básica, n. 33)

CARNEIRO, J. M.; BRITO, A. P. B.; SANTOS, M. E. A. Avaliação do desenvolvimento de crianças de uma creche através da escala de Denver II. Rev. Min. Enferm, Belo Horizonte, v. 15, n. 2, p. 174180, abr./jun. 2011. comunidade com foco na integralidade em consonância com o Sistema Único de Saúde (SUS).

Cabe ressaltar que a escassez de produções acercada temática, mesmo sabendo de todas as vulnerabilidades das crianças, constituiu uma limitação para a pesquisa.

Diante do exposto, aponta-se a necessidade da realização de novas pesquisas acerca da atuaçãode enfermagem na atenção à saúde de crianças que frequentam creches, tendo em vista a susceptibilidadedesse grupo diante de doenças e agravos que podem comprometer o pleno desenvolvimentode suas potencialidades.

CORTEZ, D. N. et al. Crianças em creche: acompanhamento do crescimento e desenvolvimento. R. Enferm. Cent. O. Min, v. 2, n. 1, p. 43-50, jan./abr. 2012.

\section{DEPARTAMENTO DE INFORMÁTICA DO SISTEMA ÚNICO DE SAÚDE DO BRASIL. DATASUS. Indicadores de Morbidade. 2010.}

ESTEVES, M. R. et al. Influências das relações intrafamiliares no comportamento de crianças que frequentam creches públicas de alfenas. Rev Gaúcha Enferm, v. 33, n. 3, p. 97-103, 2012.

FARIA, M. L.; WICHR, P. Creche, Criança e Saúde. Rev Min Enferm, Minas Gerais, v. 19, n. 1, p. 142-146, 2014.

FERNANDES, F. C.; BARBOSA, F. H. F. Ocorrência de parasitoses intestinais entre crianças da creche menino jesus do município de dores do indaiá, minas gerais. Ciência

Equatorial, v. 1, n. 1, p. 28-37, 2011.

FROTA, M. A. et al. Percepção materna em relação ao cuidado e desenvolvimento infantil. Rev. Bras. Promoc. Saude, 
Fortaleza, v. 24, n. 3, p. 245-250, jul. /set. 2011.

GABANI, F. L.; MAEBARA, C. M. L.; FERRARI, R. A. P. Pediculose nos centros de educação infantil: conhecimentos e práticas dos trabalhadores. Esc Anna Nery, Rio de Janeiro, v.14, n. 2, p. 309-317, abr. /jun. 2010.

GOLDSCHMIDT, A. I.; LORETO, E. Investigação das concepções espontâneas sobre pediculose entre pais, professores, direção e alunos de educação infantil e anos iniciais. Revista Eletrônica de Educação e Ciência, v. 11, n. 2, p. 455-470, 2012.

MINAYO, M. C. de S. Análise qualitativa: teoria, passos e fidedignidade. Cienc. $\mathbf{E}$ saudecolet, Rio de Janeiro, v. 17, n. 3, p. 621626, 2012.

MONTEIRO, F. P. M.; CAETANO, J. A.; ARAUJO, T. L. Enfermagem na saúde da criança: estudo bibliográfico acerca da avaliação nutricional. Esc Anna Nery, Rio de Janeiro, v. 14, n. 2, p. 406-411, abr./jun. 2010.

MOTTA, J. A. et al. O cuidado à criança na creche: Integração entre saúde e educação.

Revenferm UERJ, Rio de Janeiro, v. 20, n. 2, p. 771-776, dez. 2012..

MURTA, A. M. G. et al. Cognição, motricidade, autocuidados, linguagem e socialização no desenvolvimento de crianças em creche. Rev. Bras. Crescimento
Desenvolvimento Hum, v. 21, n. 2, p. 220229, 2011PEDRAZA, D. F.; QUEIROZ, D.; SALES, M. C. Doenças infecciosas em crianças pré-escolares brasileiras assistidas em creches. Cienc saúde colet, v. 19, n. 2, p. 511-528, 2014.

SANTOS, J. N.; LEMOS, S. M. A.; LAMOUNIER, J. A. Estado nutricional e desenvolvimento da linguagem em crianças de uma creche pública. Rev. Soc. Bras. Fonoaudiol, São Paulo, v.15, n. 4, p. 566$571,2010$.

SARUBBI, J. V. et al. No contexto da creche: a enfermagem e suas representações do cuidado à criança como ato educativo. Rev. Esc. Enferm. USP, São Paulo, v. 48, n. 2, p. 48-54, 2014.

SOUZA, M. H. N. et al. Avaliação do estado nutricional e da saúde de crianças e adolescentes na prática assistencial do enfermeiro. CogitareEnferm, Curitiba, v. 18, n. 1, p. 29-35, jan. /mar. 2013.

URSI, E. S. Prevenção de lesões de pele no perioperatório: revisão integrativa da literatura. [dissertação]. Ribeirão Preto (SP): Universidade de São Paulo, p. 130, 2005.

VASCONCELOS, R. M.; TANCREDI, R. C. P.; MARIN, V. A. Políticas e normativas aplicadas às creches municipais do Rio de Janeiro. Cienc saúde colet, Rio de Janeiro, v. 18, n. 11, p. 3281-3290, 2013. 